\title{
Assuaging nutritional complexity: a geometrical approach
}

\author{
Stephen J. Simpson* and David Raubenheimer \\ Department of Zoology and University Museum of Natural History, University of Oxford, South Parks Road, \\ Oxford OX1 3PS, UK
}

\begin{abstract}
We have introduced a framework that enables the identification of the important elements in complex nutritional systems, and the quantification of the interactions among them. These interactions include those among the multiple constituents of the ingesta, as well as between behavioural (ingestive) and physiological (post-ingestive) components of nutritional homeostasis. The resulting descriptions provide a powerful means to generate and test hypotheses concerning the mechanisms, ecology and evolution of nutritional systems. We provide an overview of the key concepts involved in our scheme, and then introduce four examples in which the framework is used to develop and test hypotheses. In the first example we use comparative methods based on a data set of 117 insect species to test a prediction about the relationship between evolving an association with bacterial endosymbionts and the composition of the optimal diet. Second, using two species of locusts (a grass specialist and a generalist), we consider the relationship between an animal's diet breadth and the decision rules employed when feeding on foods containing suboptimal protein : carbohydrate values. Third, we introduce a mathematical model that predicts the dose-response properties of gustatory systems in the context of nutritional homeostasis. Finally, we consider the interaction between tannic acid and macronutrient balance in the diet of locusts.
\end{abstract}

Nutrient balancing: Geometric models of nutrition: Insects: Feeding behaviour

\begin{abstract}
Nutritional regulation by an animal represents the integrated outcome of a highly complex set of interacting processes, operating over a range of scales of organization. Central to these processes is the acquisition and allocation of the more than thirty different nutrient molecules required for survival and reproduction. These nutrient molecules come packaged in various ratios and concentrations within foods, which may also contain an array of non-nutritive molecules, some of which are harmful. Foods are distributed across space and time, and finding, eating, digesting, assimilating and utilizing them involves ecological and physiological costs and compromises. Further, the nutritional environment is frequently highly variable, at a series of different scales; spatial variation occurs from mouthful-to-mouthful, patchto-patch and locality-to-locality, while temporal variation spans physiological, developmental and evolutionary time. Equally variable are the requirements of the animal, which are multidimensional and change quantitatively and qualitatively as an individual grows, develops, becomes reproductively active, then senesces.
\end{abstract}

Evolution has clearly achieved viable solutions to these various challenges; but how do we as scientists deal with studying and interpreting the multidimensional, interactive nature of nutrition within an organismal framework? The short answer is that, generally, the multidimensional and interactive nature of nutrition has not adequately been taken into account. For example, in behavioural ecology, it is frequently assumed that a single food property, usually its energy content, is pre-eminent (Stephens \& Krebs, 1986; Hughes, 1993). Nutritional ecologists, on the other hand, have concentrated on another food characteristic, $\mathrm{N}$ content (for example, see McNeill \& Southwood, 1978; Mattson, 1980; White, 1993). Those workers concerned with elucidating the mechanisms controlling macronutrient selection have tended to manipulate dietary levels of one focal component, and have not dealt fully with the commensurate changes in other components (for examples, see Simpson \& Raubenheimer, 1997).

The univariate approach has proven valuable in some circumstances, but difficulties arise when nutritional 
regulation involves several nutrient groups and when the foods selected by an animal are not all similar in composition (i.e. most of the time). In such cases, the system can be understood only by taking into account all the relevant nutrient dimensions. In recognition of this factor, proponents of the univariate approach have attempted to encompass in their models food properties other than that judged to be the pre-eminent one, by including them as constraints on the primary nutritional currency (for example, see Pulliam, 1975; Belovsky, 1990). While this approach might be adequate for cases where a single nutritional currency interacts with deleterious substances such as plant toxins, it engenders an unwelcome element of arbitrariness when two or more nutrients interact: which is to be considered the currency, and which the constraint? The answer to this problem is not fixed, but is dynamically dependent on current circumstances (e.g. the animal's current nutritional state in relation to requirements, relative availability of the two nutrients etc.). To capture such aspects, a different methodological framework is required.

An alternative approach is to make no a priori judgements about the relative importance of different food properties, nor of an animal's nutritional requirements, but rather to begin by treating all food components as equal and allowing the animal to indicate how it prioritizes their ingestion and utilization. Such an approach treats the interactions among food components as the primary variable, and enables the construction of models of nutrition that are founded on the biological characteristics of the organisms, rather than on our a priori expectations. It also provides an opportunity to detect context-dependent responses of animals to food components, i.e. responses whose expression depends on other components of the nutritional milieu.

A suitable experimental and theoretical approach would be one that: (a) allows measurement of an animal's multiple nutritional requirements, and hence identifies the composition of a nutritionally-balanced food and how much of this food should optimally be eaten over a given period of time; (b) enables measurement and interpretation of the trade-offs reached between overeating some nutrients and undereating others when available foods are nutritionally imbalanced; (c) provides a means of integrating the study of feeding behaviour with that of post-ingestive physiology and metabolism; (d) allows integration across levels of biological analysis, including causation, development, ecology and evolution.

It was with these aims that we have developed a class of geometric models of nutrition (Raubenheimer \& Simpson, 1993, 1994, 1995, 1996, 1997, 1998, 1999; Simpson \& Raubenheimer, 1993a, 1995, 1996, 1997, 1999a,b; Simpson et al. 1995). Our major study systems have been various species of herbivorous insects, notably locusts, but we have also recently demonstrated the generality of our models by applying them to published data on mammals and birds (Raubenheimer \& Simpson, 1997; Simpson \& Raubenheimer, 1997, 1999a).

Our aim in the present paper is briefly to review the geometric models and to illustrate some applications to evolutionary, ecological and mechanistic questions using data for insects, some of which are as yet unpublished. Further discussion and justification of the models them- selves may be found in the references mentioned earlier, while full details of the new experiments will be published elsewhere over the coming year.

\section{The geometrical approach}

\section{Representing requirements: functional targets in nutrient space}

An animal is depicted as existing within a multidimensional nutrient space, where each axis represents a nutrient. Dimensions for non-nutrient compounds found in foods may also be included. Within nutrient space lie regulatory targets, which have evolved through natural selection on the differential consequences of the amounts and balance of nutrients ingested. Targets may be considered either as static points, integrated over a given period in the animal's life, or as dynamic trajectories.

The hierarchy of processes that comprise nutritional regulation will involve several target points. The most fundamental of these target points, the nutrient target, represents the combined requirements of the animal's tissues. A component of the nutrient target, the growth target, represents the optimal nutrient allocation to somatic, reproductive and storage growth. The remaining nonstructural component of the nutrient target comprises the optimal nutrient requirements for supplying energetic needs. Reaching the nutrient target involves locating, selecting and ingesting food. The intake target is the combination of nutrients whose ingestion provides an animal with nutrients to its tissues at the optimal rate and balance. The intake target will exceed the nutrient target in its various nutrient dimensions because of the inevitable loss of nutrients in converting food to meet tissue needs.

\section{Representing foods: nutritional rails}

Foods are mixtures of various nutrient and non-nutrient compounds, and as such may be represented as lines radiating out from the origin into nutrient space, at angles that are defined by the ratio of their constituents. If an animal is confined to eating only one food it is forced to ingest the ratio of nutrients contained in that food. The animal cannot change trajectory within nutrient space without switching to another food or differentially utilizing nutrients post-ingestively. To capture the idea of being confined to a given trajectory we have used the term rail for food lines.

\section{The relationship between foods and requirements}

The vector in nutrient space from the point representing an animal's current nutritional state to the intake target determines which food or foods need to be ingested if the animal is to reach its intake target. An optimal food is the one that enables the animal to move directly to the intake target, thus simultaneously achieving its multiple nutrient requirements.

There are two ways in which an animal might reach its intake target over a given period: (a) by selecting the optimal food, if it exists, from among the cafeteria of food items available in its environment, or (b) by mixing its 
intake from two or more suboptimal but nutritionally complementary foods. Complementary foods are ones whose rails delimit an area in nutritional space that includes the intake target.

\section{Nutritional compromises}

If the animal has only non-complementary suboptimal foods available, it will not be able to reach its intake target. As a result, the animal will have to compromise between eating some nutrients in excess and undereating others, relative to the intake target. The point of balance reached is determined by the relative weighting of the animal's regulatory systems for the nutrients involved, and hence would be expected to reflect their relative functional importance to the animal. When measured across a range of suboptimal food rails, these points of compromise form intake arrays that define a more general rule of compromise. Analysis of these arrays allows the manner in which regulatory systems weight undereating and overeating different nutrients to be quantified (Raubenheimer \& Simpson, 1997).

\section{Measuring the intake target}

Estimating the location of the intake target is a primary aim of any nutritional study. There are three ways of doing this: (a) by erecting a performance axis (representing fitness consequences in terms of mortality, development rates etc.) onto a nutrient space, and identifying the highest point on the resulting performance landscape; (b) by challenging the animal to defend a point of intake in nutrient space, and assuming that such points of homeostasis have been aligned with fitness maxima over evolutionary time; (c) by reconstructing the intake target from measurements of growth, respiration and wastage.

We have used a combination of all three of the previously mentioned techniques for locusts in the context of protein and carbohydrate regulation, and found closely similar outcomes (Raubenheimer \& Simpson, 1993, 1997; Simpson \& Raubenheimer, 1993a,1999b). We have challenged locusts to defend their intake in carbohydrate-protein space by using three different experimental regimens (Fig.1).

Pairs of complementary foods. When locusts (Locusta migratoria) were provided with one of four complementary food pairings (protein: carbohydrate content; 28:14 or $14: 7 v$. either $14: 28$ or $7: 14$ ) they adjusted the amount and ratio of the two foods eaten to maintain a constant point of protein and carbohydrate intake (Chambers et al. 1995; Fig. 1(a)).

Food dilution. When locusts were given one of five foods with an approximately optimal protein-digestible carbohydrate value $(1: 1)$, but diluted up to fivefold with indigestible cellulose (protein : digestible carbohydrate content; $35: 35,28: 28,21: 21,14: 14$ or $7: 7$ ), they adjusted their consumption across all dilutions to maintain a constant point of nutrient intake (Raubenheimer \& Simpson, 1993; Fig. 1(b)).

Foodfrequencies. When two complementary foods (protein: digestible carbohydrate content; $31: 11$ and $7: 35$ ) were provided in relative abundances of one + three, two + two or three + one dishes of one $v$. the other food type, locusts precisely defended a point of protein : carbohydrate intake by adjusting their distribution of consumption between dishes (ST Behmer, D Raubenheiner and SJ Simpson, unpublished results; Fig. 1(c)). This experiment was designed as part of a programme in which spatial complexity is introduced to our nutritional models in the form of various frequencies and spatial distributions of foods.

These remarkable feats of homeostasis were found to extend to regulation of salt $v$. macronutrient intake (Trumper \& Simpson, 1993). Re-analysis of data from rats and hens showed that they too defend a point of intake in carbohydrate-protein space (Raubenheimer \& Simpson, 1997; Simpson \& Raubenheimer, 1997).

\section{Measuring rules of compromise}

As is the case for the estimation of the location of the intake and other targets, the nature of rules of compromise is a matter for experimental measurement, not a priori assumption. Experimental designs simply involve providing animals with one of an array of suboptimal foods and measuring intake. We have demonstrated various rules of compromise for salt, protein and carbohydrate intake in locusts, rats and hens (see Raubenheimer \& Simpson, 1997). The more different species that are investigated, the greater the likelihood of uncovering fundamental relationships between targets, rules of compromise and ecological and life-history characteristics (Simpson \& Raubenheimer, 1993a).

\section{Incorporating time: the dynamics of nutritional regulation}

We have in the preceding discussion considered targets and rules of compromise as patterns integrated over a stipulated time period. In reality, targets move through nutrient space at a range of time scales, both within the life of an individual over physiological and developmental time, and across generations through evolutionary time. They can thus be viewed as trajectories in nutrient space. Similarly, rules of compromise can be viewed as unfolding rather than static patterns. This approach enables the explicit representation of time in our models. Examples are given in Raubenheimer \& Simpson (1997).

\section{Integrating pre- and post-ingestive regulatory systems}

The interactions occurring between feeding behaviour and post-ingestive physiology provide some of the most important challenges in the study of nutrition. While intake is constrained by the composition of available foods, animals are able to range more widely in nutrient space by differentially utilizing ingested nutrients. Hence, the growth target may be reached from a range of intake points in nutrient space. Locusts show highly efficient defence of a growth target in protein-carbohydrate space, as do rats and hens (Raubenheimer \& Simpson, 1997; Fig. 2). In locusts, ingested protein and carbohydrate is assimilated with high efficiency up to their growth target levels and, thereafter, any excess ingested $\mathrm{N}$ is excreted, while excess $\mathrm{C}$ is respired (Zanotto et al. 1993, 1994, 1997). 
(a) Diet selection

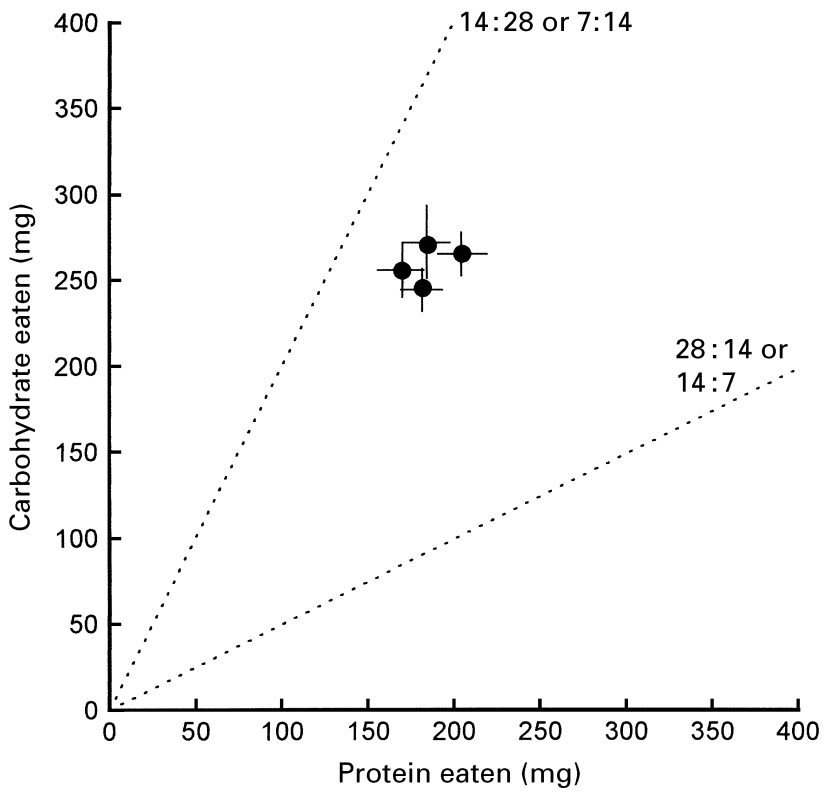

(b) Nutrient dilution

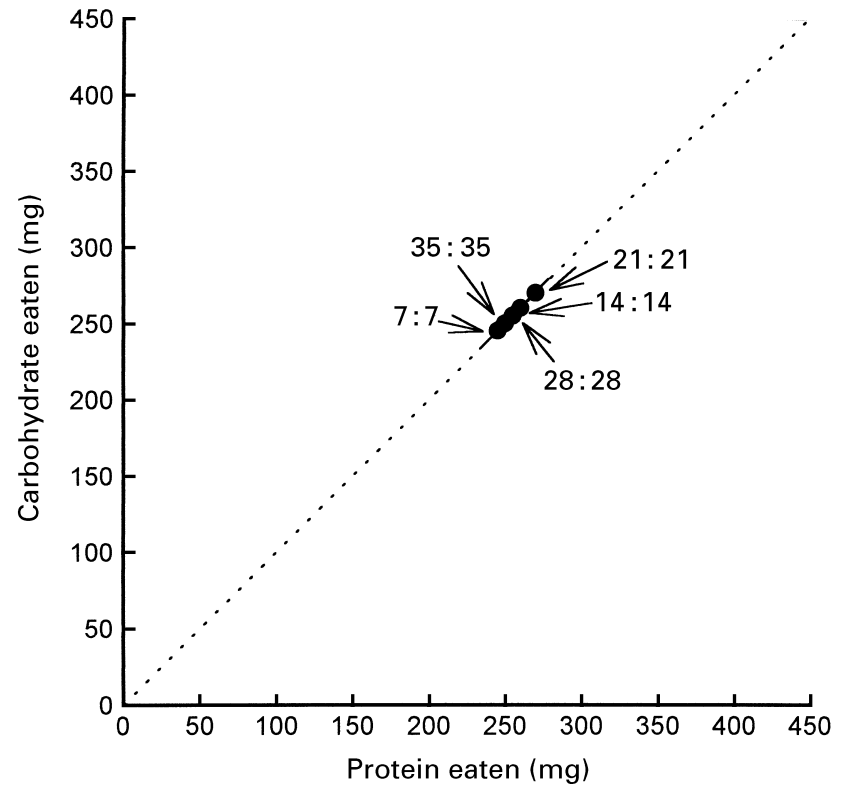

(c) Food frequency

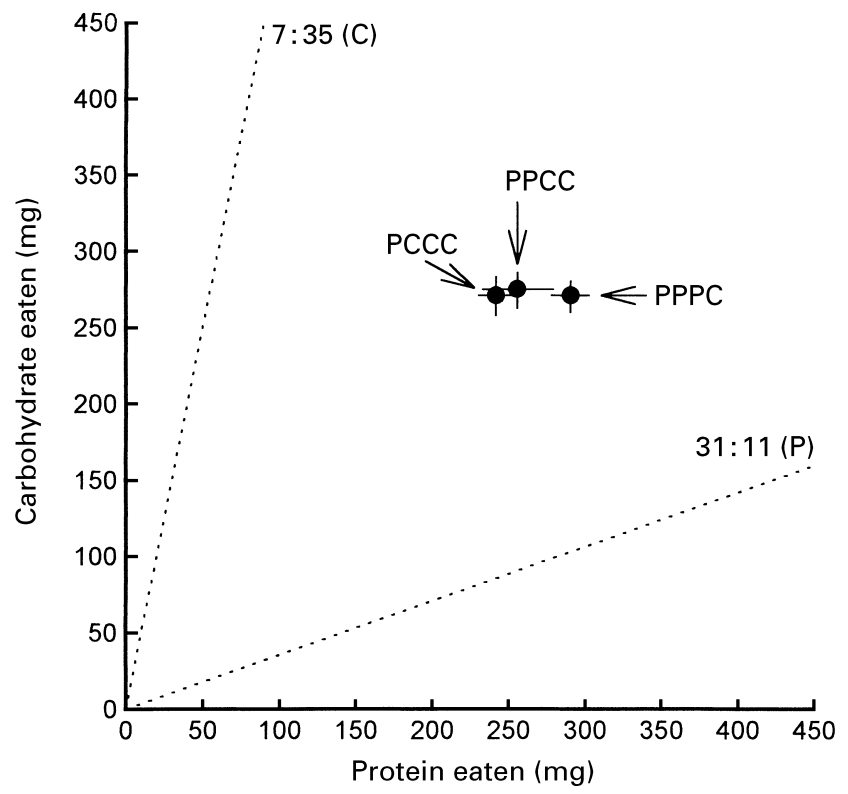

Fig. 1. Three experiments indicating defence of a point of intake in a carbohydrate-protein nutrient plane. (a) Locusts (Locusta migratoria) were provided with one of four food choices (protein : carbohydrate content; $14: 28$ or $7: 14$ v. 28:14 or 14:7) and altered relative amounts eaten from the two foods, thus reaching the same place in nutrient space. (From Chambers et al. 1995.) (b) Locusts were provided with one of five foods with a protein : digestible carbohydrate value of $1: 1$, but diluted to various degrees with indigestible cellulose. Those locusts receiving food with a protein : digestible carbohydrate content of $7: 7$ ingested five times more food than those on a food with a corresponding value of $35: 35$, thus achieving the same intake of both protein and digestible carbohydrate. (From Raubenheimer \& Simpson, 1993.) (c) Locusts were given four food dishes with a protein : carbohydrate content of 7:35 (food C), or 31:11 (food P). The two food types were provided at different frequencies (all four dishes contained $\mathrm{C}$, all four contained $\mathrm{P}$, or two contained $\mathrm{C}$ and two $\mathrm{P}$ ). Locusts adjusted the amounts eaten from each dish and defended nutrient intake (ST Behmer, D Raubenheimer and SJ Simpson, unpublished results).

We have begun to consider the dynamic interactions between pre- and post-ingestive mechanisms regulating intake and utilization of macronutrients in a class of models based around the marginal value theorem (Raubenheimer \&
Simpson, 1996, 1998). These models deal with the relationship between rates and efficiencies at various stages of nutrient processing, as well as the interactions among different nutrient groups. 


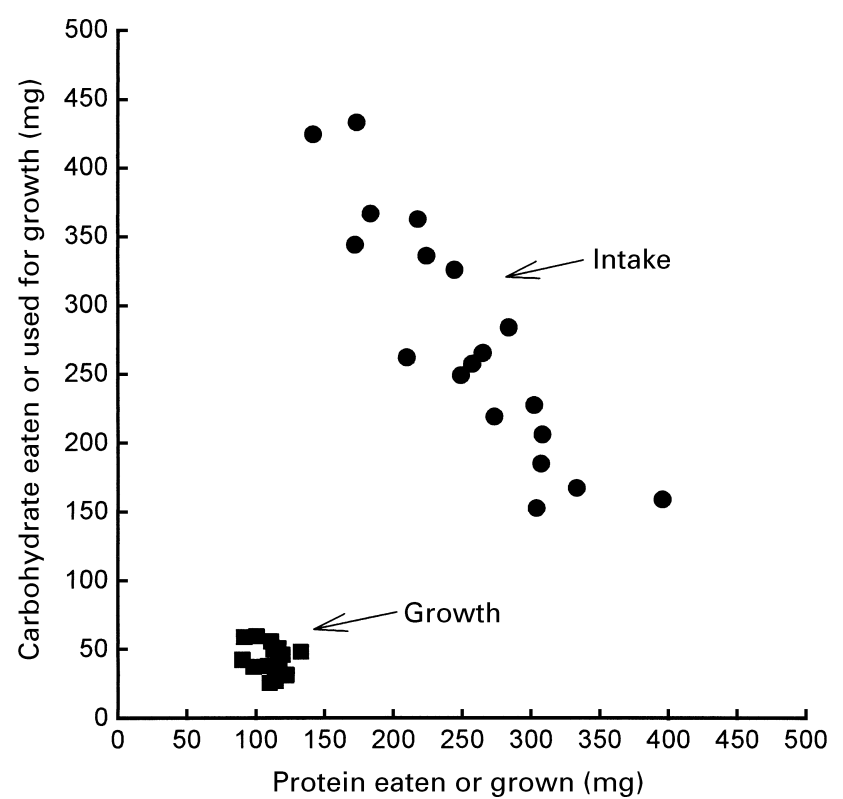

Fig. 2. Points of intake and growth from an experiment in which locusts were provided with one of nineteen foods varying in protein and digestible carbohydrate content. Note how although intake was constrained to particular values for protein:carbohydrate by the nature of the foods, locusts nevertheless achieved highly similar growth through differentially utilizing ingested nutrients. The foods contained only trace lipid. Carbohydrate-derived growth includes both lipid and structural carbohydrate, such as cuticle. (From Raubenheimer \& Simpson, 1993.)

\section{Applying the framework: deriving and testing hypotheses}

To this point we have introduced the basic components of our geometrical framework of nutrition. While it is fundamentally a technology for describing aspects of nutritional systems that derive from the interactions among the component parts (Raubenheimer \& Simpson, 1999), our primary aim is to use such descriptions to explore broader evolutionary, ecological and mechanistic issues. In the final part of the present paper we illustrate some applications, using examples from insects.

\section{Evolutionary hypotheses: selective bases for evolutionary shifts in intake targets}

Insects provide an excellent opportunity to explore evolutionary shifts in intake targets, given the extraordinary range of food sources exploited by the 1000000 described (let alone the perhaps 10000000 as yet undescribed) species. In a survey of the diets of 117 species of insect we found that the protein: carbohydrate value of the foods that sustained good development varied from 100:0 to 6:94. Such data were used to test a specific hypothesis regarding the physiology and life history of the different species (Simpson \& Raubenheimer, 1993a).

Over recent years there has been considerable interest in the nutritional role played by micro-organisms housed within specialized cells (mycetocytes) of some insects species. There is evidence that such microbial associations play an important role in enhancing the efficiency of nitrogenous nutrition of the insect host (Douglas, 1994). It might be predicted, therefore, that insect species possessing mycetocyte symbionts would have an intake target with a lower dietary protein : carbohydrate value than would those species lacking the microbial association.

In testing such an hypothesis it is not valid to treat species as statistically-independent points, since species may share the same traits by descent (Harvey \& Pagel, 1991). Overcoming such a problem involves controlling for phylogenetic relationships between species in the analysis. Within the 117 species of insect there were eight nodes in the phylogeny that provided independent contrasts between species possessing and lacking mycetocyte symbionts (Fig. 3). In each of these eight cases, species possessing symbionts had a lower dietary protein : carbohydrate value than those without symbionts. We are presently experimentally manipulating symbiont levels in cockroaches, and assessing the effects such treatments have on the position of the intake target and also on rules of compromise.

\section{Two ecological hypotheses}

Rules of compromise in specialist and generalist feeders. In an early experiment we derived intake arrays for the African migratory locusts, L. migratoria, fed one of an array of 25 foods varying systematically in protein and carbohydrate content (Raubenheimer \& Simpson, 1993). The resulting points of protein and carbohydrate intake formed a pattern in which locusts on average moved to the geometrically closest point on their food rail to the intake target. By following this closest distance rule the animal is minimizing the sum of undereating one nutrient and overeating the other, irrespective of which of the two nutrients happens to be in excess or deficit (Raubenheimer \& Simpson, 1997).

We next compared L. migratoria concurrently with the related desert locust, Schistocerca gregaria (Fig. $4(a, b))$. Both species defended the same value for protein : carbohydrate intake in choice tests. However, while L. migratoria, as expected, observed the closest distance rule when given suboptimal foods, $S$. gregaria followed the equal distance rule, moving along their food rail to the point where the sum of protein and carbohydrate was the same as at the intake target. In so doing, Schistocerca ingests a greater total amount of nutrients on suboptimal foods than Locusta, but incurs greater error with respect to the intake target.

The difference in the rules of compromise between the two species could be explained in terms of their respective ecologies. Schistocerca will eat a wide range of plant species, which possibly means that it encounters a wider variety of food nutrient compositions than does the grassspecializing Locusta. If so, there will be a greater probability that the generalist will subsequently encounter an edible plant with a complementary imbalance to the present food, allowing the ingested excess from the current food to balance the deficit in the subsequent one, hence converting excess into useful nutrient. 


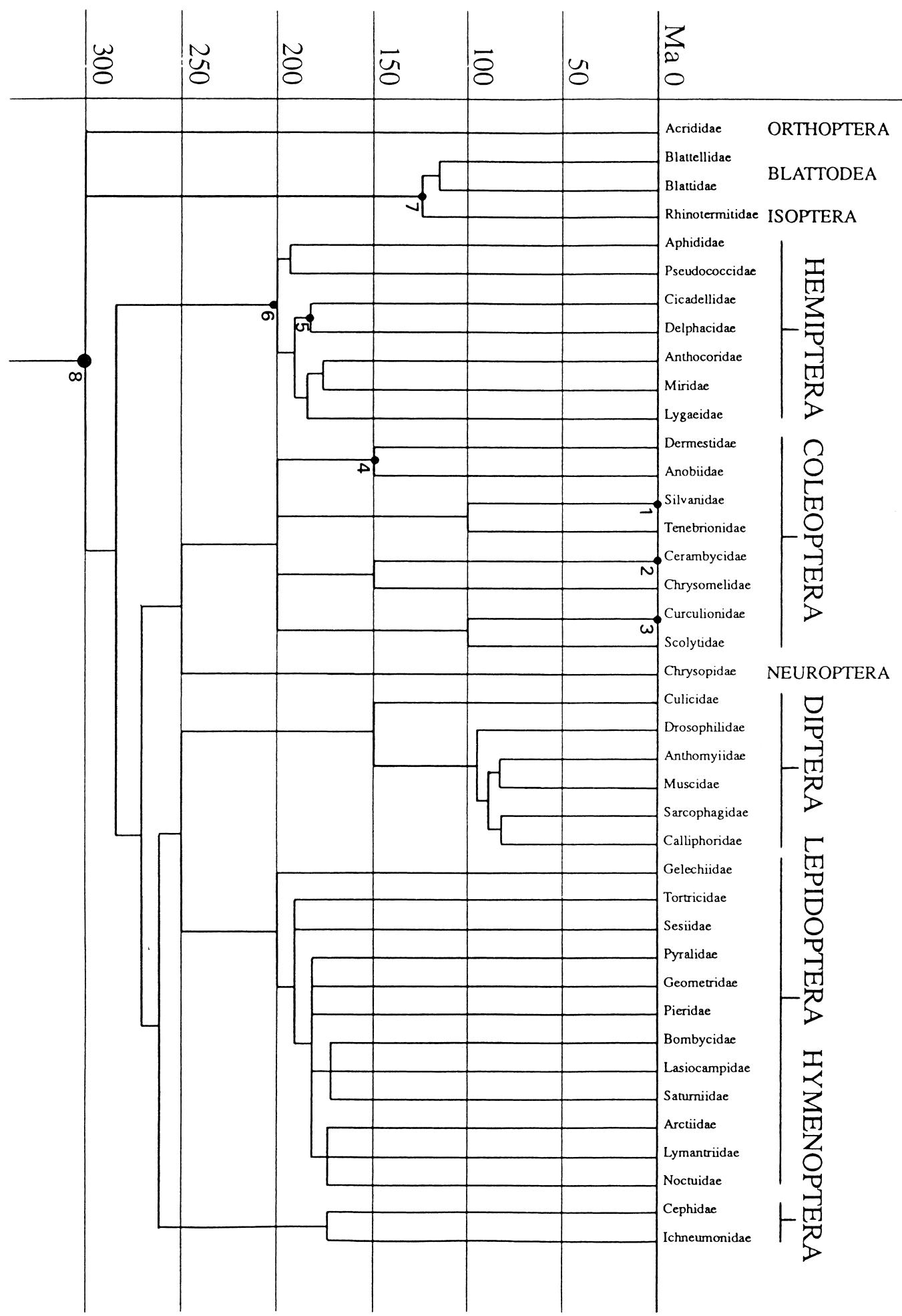

Fig. 3. The phylogeny used in the comparative analysis exploring the association between the presence of mycetocyte symbionts in insects and protein (and amino acids): digestible carbohydrate in their diet. The analysis included 117 insect species from forty families. Numbered nodes are those where phylogenetically-independent contrasts could be made. Node 8 is a point of independent contrast if the single species of termite was removed from the phylogeny. At each node there was a reduction in the protein: carbohydrate in the diet, as predicted, with changes in the value $P /(P+C) \times 100$, where $P$ and $C$ are $\%$ dietary protein and digestible carbohydrate respectively of $-34 \cdot 0,-3 \cdot 3,-31 \cdot 4,-27 \cdot 7,-42 \cdot 0$, $-32 \cdot 0,-23 \cdot 2$ and $-12 \cdot 3$ for nodes $1-8$ respectively. (From Simpson \& Raubenheimer, 1993a.) 
(a) Gregarious Locusta

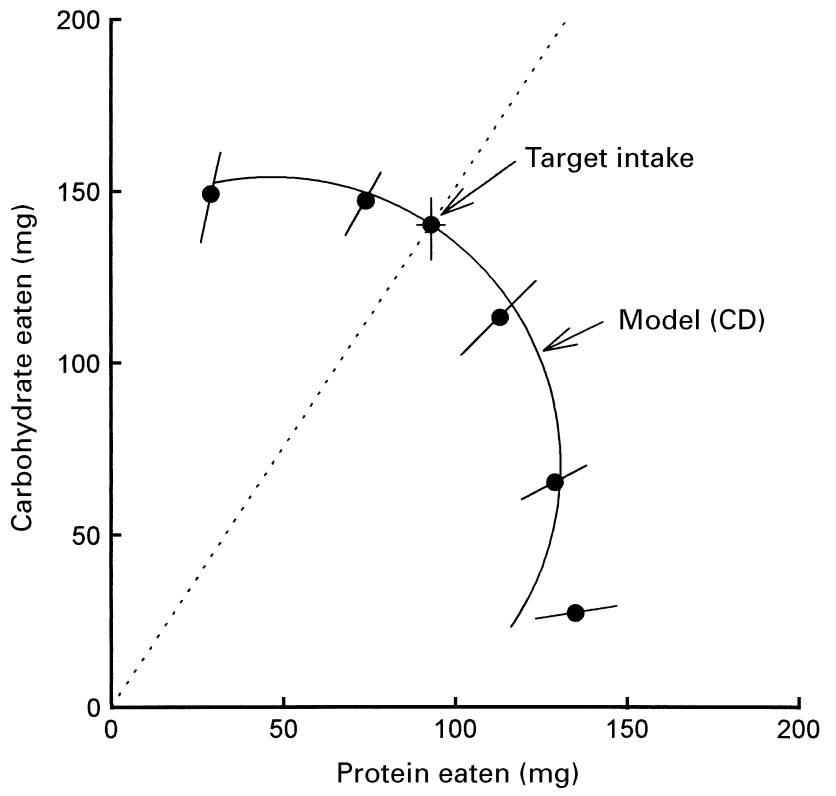

(c) Solitarious Schistocerca

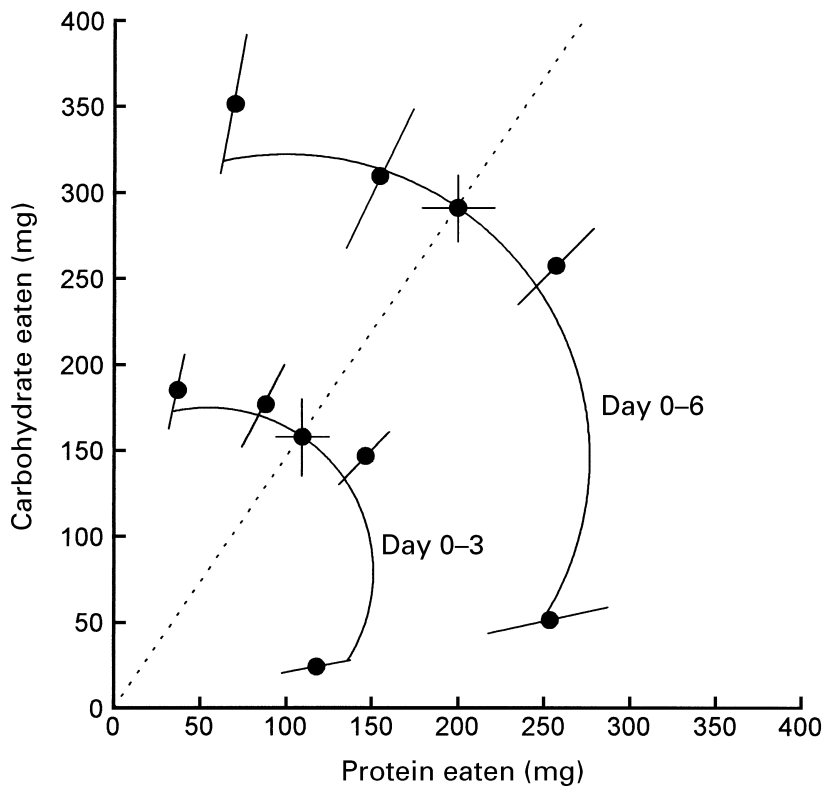

(b) Gregarious Schistocerca

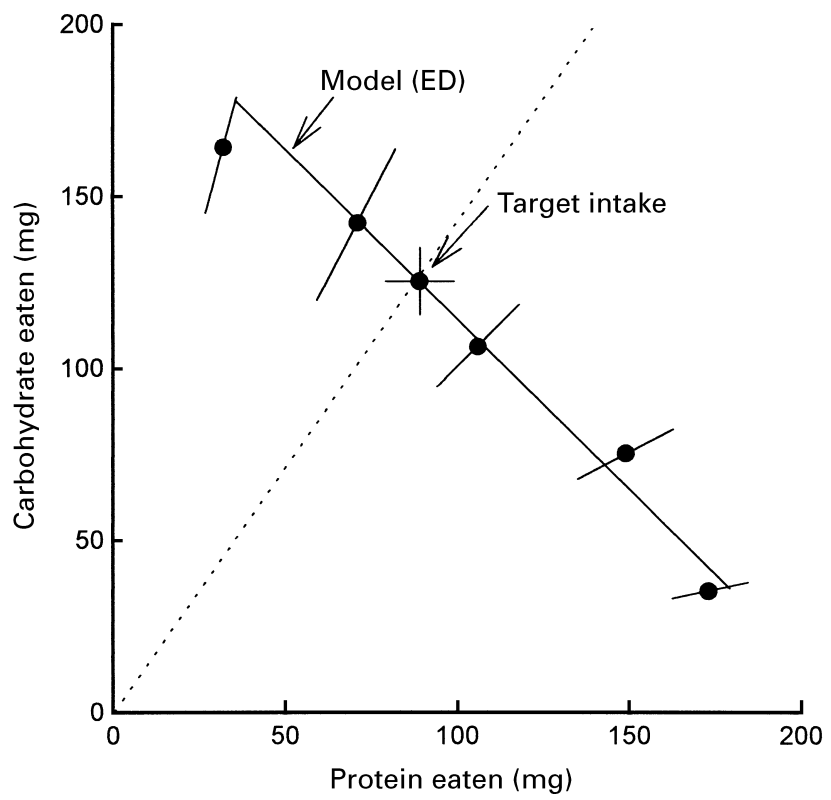

(d) Gregarious Schistocerca

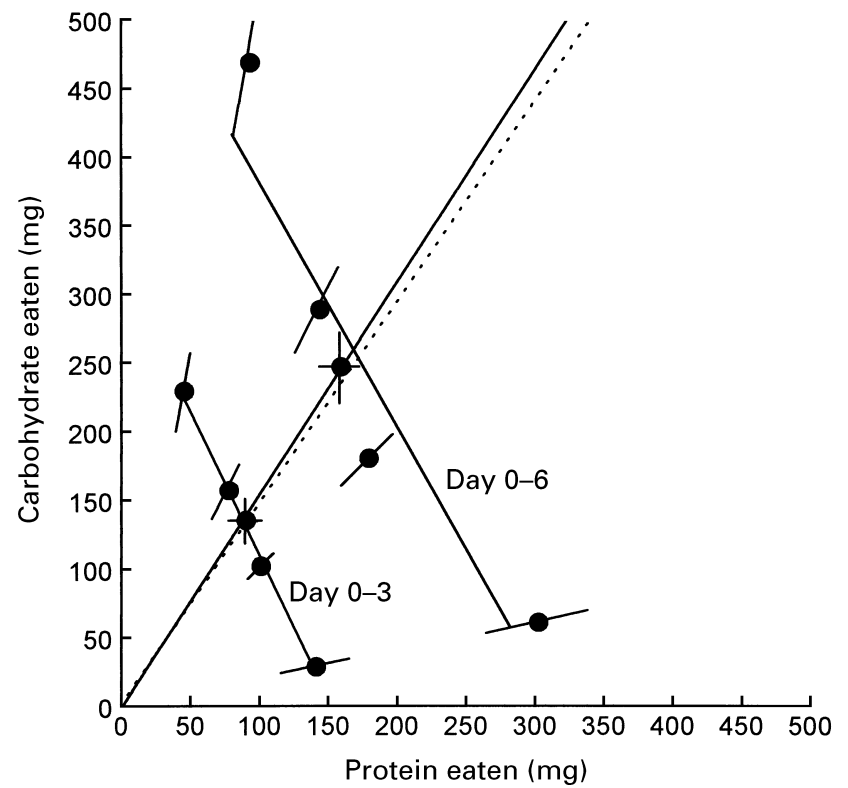

Fig. 4. Data from comparative studies of two locust species, Locusta gregaria (a grass specialist) and Schistocerca gregaria (a broad generalist). (a) and (b), These plots show points of intake in an experiment where locusts were either provided with a single food (protein: carbohydrate content; $7: 35,14: 28,21: 21,28: 14$ or $35: 7$ ) or the opportunity to select their intake. Note how, when forced to feed on a suboptimal food, the food specialist followed the closest distance $(C D)$ rule, thus minimizing error with respect to the unattainable intake, while the generalist species maximized nutrient intake (equal distance (ED)). (c) and (d), These plots compare in a separate experiment the responses of the gregarious (swarming) phase of Schistocerca gregaria and the solitarious phase of the same species. Note how the solitarious phase follows the CD rule of compromise, which perhaps reflects its more specialized diet relative to the migratory gregarious phase (D Raubenheimer and SJ Simpson, unpublished results; SJ Simpson, D Raubenheimer, G Wright and ST Behmer, unpublished results).

We might tentatively suggest, therefore, that species that experience a relatively narrow range of food nutrient compositions are more likely to follow the error minimization rule, while species that feed on a wide range of food types are more likely to be nutrient intake maximizers (Raubenheimer \& Simpson, 1999).
As we have seen previously, one potential difficulty in testing such an hypothesis is that phylogenetic effects confound interpretation (Harvey \& Pagel, 1991). While this problem can be dealt with statistically through the use of comparative methods involving phylogeneticallyindependent contrasts, data from large numbers of species 
representing numerous higher taxa are required, but are not as yet available. An alternative, and in some ways ideal, solution would be to have genetically-identical forms of the same species that exhibit different feeding strategies.

The desert locust offers just such an opportunity. It demonstrates phase polymorphism (Uvarov, 1966; Simpson et al. 1999). Under low population densities it exists in the solitarious form, which, because of the desert conditions in which it lives, tends to be restricted to eating a small number of species of plants. At high population densities, however, the insect changes into the highly-polyphagous swarming gregarious form. Swarms and bands of gregarious locusts move considerable distances and each individual is likely, therefore, to experience a wider range of food compositions, and is also subject to greater competition for food from other locusts than the solitarious form.

It might be expected, therefore, that the gregarious form would be a nutrient maximizer, while the solitarious form would be predicted to demonstrate error minimization. Recent data from an experiment in which we compared the two phases in the laboratory meet these predictions (SJ Simpson, D Raubenheimer, G Wright and ST Behmer, unpublished results). Both phases defended a closely similar intake target protein: carbohydrate value, but their rules of compromise differed. While the gregarious form demonstrated a pattern of intake consistent with the equal distance rule, data from solitarious-phase insects fitted the closest distance rule (Fig. 4(c,d)).

Nutrient-allelochemical interactions. An important part of the nutritional milieu of many animals is the various nonnutrient chemicals. Plant secondary metabolites are one obvious example. Just as the behavioural and physiological responses to a given nutrient are contingent on the other nutrients present, so too might they depend on levels of nonnutrients, especially when such compounds are harmful. Common components of plant tissues are hydrolysable tannins, which are able to bind and precipitate proteins (Bernays \& Chapman, 1994; Schoonhoven et al. 1998). Accordingly, it has been argued that such compounds act to reduce the available protein content of the diet, through complexing with proteinase enzymes, their substrates, or both (Van Hoven, 1984; Mole \& Waterman, 1987). From this information it might be predicted that tannins would have the greatest detrimental effect when protein levels in the foods are low.

Following on from earlier work (Raubenheimer \& Simpson, 1990; Raubenheimer, 1992), we have recently undertaken an experiment in which locusts were provided with one of five foods (protein : carbohydrate content; $7: 35$, $14: 28,21: 21,28: 14$ or $35: 7$ ) containing one of four levels of tannic acid (g/kg diet; 0, 33, 67 or 100). Survival (Fig. 5), intake (Fig. 6), and efficiency of $\mathrm{N}$ utilization were all strongly contingent on the nutrient balance of the food. When foods contained an approximately optimal ratio and amount of protein: carbohydrate $(21: 21)$ there was no discernible effect of adding even $100 \mathrm{~g}$ tannic acid $/ \mathrm{kg}$ diet. However, as the foods became increasingly nutritionally unbalanced, deleterious effects became rapidly more prominent, especially on foods with low protein: carbohydrate values (SJ Simpson and D Raubenheimer, unpublished results). Hence, the data supported the prediction to some degree, but also provided new insights. It was not predicted from standard dogma that performance should also have been affected when diets contained a higher than optimal protein: carbohydrate value. Investigation of $\mathrm{N}$ utilization plots (see Raubenheimer \& Simpson, 1994) suggested an extra effect in which tannic acid influenced the acidity of the food and induced enhanced production of $\mathrm{NH}_{3}$, and hence loss of $\mathrm{N}$, as part of acid-base regulation.

\section{Mechanistic hypotheses: the design of taste systems}

Targets move, tracing trajectories through nutrient space at a range of time scales, both within the life of an individual over physiological and developmental time, and across generations through evolutionary time. The functional aim of an animal's regulatory systems is to track these trajectories across each time scale, taking advantage of information provided by feedbacks offering differing degrees of temporal resolution, from short-term metabolic feedbacks and learning through to natural selection.

Tracking the intake target requires two sources of information, the first regarding the composition of the food and the second the nutritional state of the animal. The major source of information regarding the composition of the food comes from food-related sensory cues, principally taste. The design features of the gustatory system (including both peripheral and central neural components) ought, therefore, to reflect feedbacks operating across all time scales. In fact, three predictions can be made about taste systems: (a) gustatory sensitivity will have evolved to nutrients whose intake is specifically regulated; (b) the taste system will show default dose-response characteristics that aid an animal in balancing its intake of such nutrients; (c) these dose-responses will be subject to modulation through more current feedback mechanisms (Simpson \& Raubenheimer, 1996).

The first of these predictions is met by all organisms, from bacteria to mammals (for example, for insects, see Chapman, 1995). Regarding the second prediction, we have, within the context of the geometric framework, developed a mathematical model for the response properties of gustatory systems that would allow an animal to demonstrate several adaptive behavioural responses: (a) to mix its intake of two or more complementary foods to provide the optimal concentrations and balance of nutrients in its diet; (b) to eat predominantly from the optimal food, if it is available; (c) to ingest most of the food that is closest to being optimal if all available foods are suboptimal and noncomplementary in composition. We have tested this model using locusts in several ways, and found that the results meet the predictions in each case (for review, see Simpson \& Raubenheimer, 1999b).

Our mathematical model describes features of the taste system that are established over evolutionary time, reflecting ancestrally-prevailing ecological circumstances and average nutrient requirements. Such a system will enable an animal to locate and defend its optimal food composition, but cannot regulate the amount of the optimal food eaten to reach the intake target, nor compensate for nutritional perturbation within the life of an animal. It is here that shortterm metabolic feedbacks and learning come into play, 

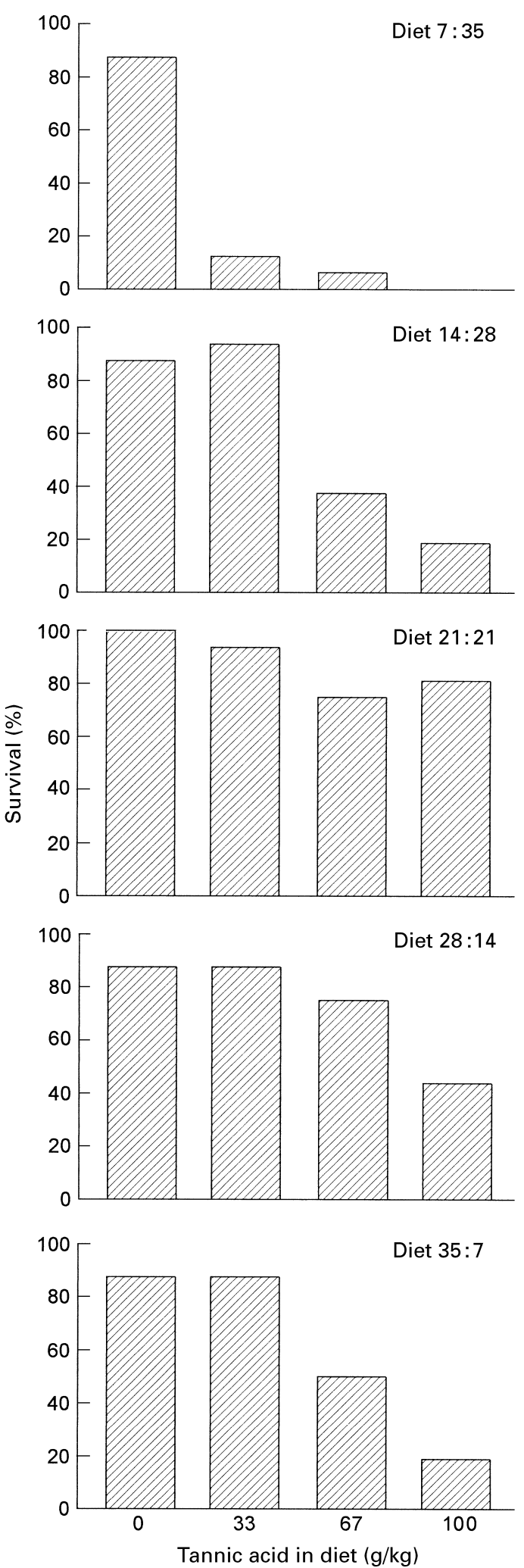

Fig. 5. Results from an experiment in which locusts were provided with one of five foods (protein : carbohydrate content; $7: 35,14: 28,21: 21$, $28: 14$ or $35: 7$ ) containing $0,33,67$ or $100 \mathrm{~g}$ tannic acid $/ \mathrm{kg}$. Note how the effect on survival of adding tannic acid to the food was strongly contingent on the nutritional balance of the food. At an approximately optimal protein : carbohydrate content $(21: 21)$ there was no measurable effect on survival, yet mortality increased markedly as the diet became nutritionally unbalanced, especially towards a low protein : carbohydrate value (SJ Simpson and D Raubenheimer, unpublished results). 


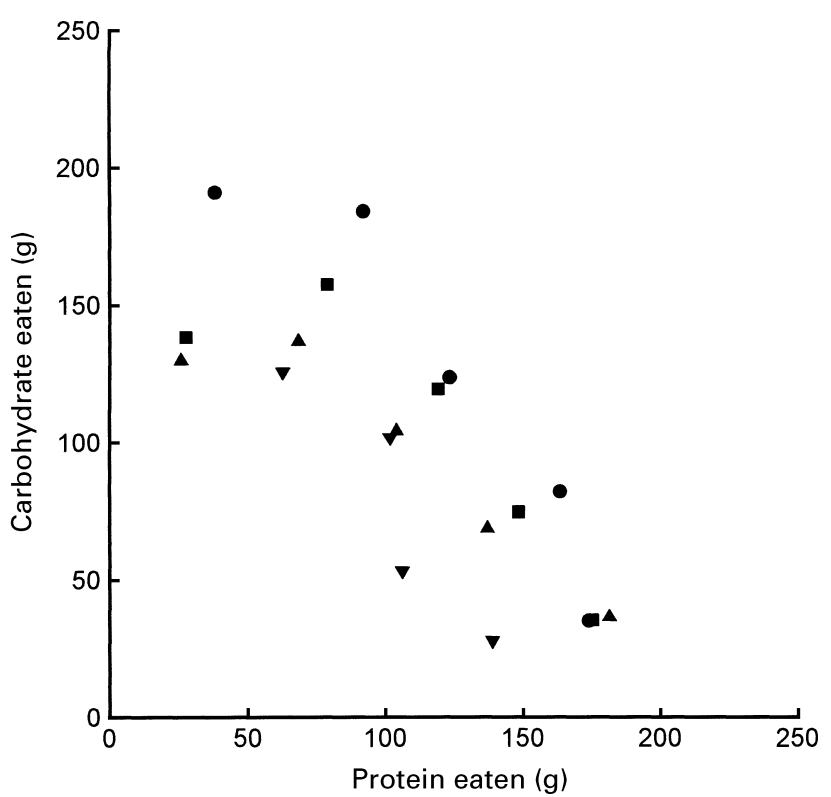

Fig. 6. Data showing the effect of tannic acid in the food on nutrient intake in surviving locusts from the experiment shown in Fig. 5 in which locusts were provided with one of five foods (protein : carbohydrate content; 7:35, $14: 28,21: 21,28: 14,35: 7$ ). Intake was affected as a function of the protein : carbohydrate value in the food. $(\boldsymbol{\nabla}), 100 \mathrm{~g}$ tannic acid (TA)/ $\mathrm{kg}$ (note that there is no such symbol for diet 7:35 since no insects survived); $(\mathbf{\Delta}), 67 \mathrm{~g} \mathrm{TA} / \mathrm{kg}$; $(\boldsymbol{\square}), 33 \mathrm{~g} \mathrm{TA} / \mathrm{kg} ;(\boldsymbol{\bullet})$, no TA. (SJ Simpson and D Raubenheimer, unpublished results.)

providing the finest scale of temporal feedback (Simpson \& Raubenheimer, 1996). Insects possess an abundance of such responses (Simpson \& Simpson, 1992; Simpson \& Raubenheimer, 1993b; Bernays, 1995; Simpson et al. 1995), and these responses enable food selection and consumption to respond rapidly to changing needs (for example, see Chambers et al. 1995).

\section{Summary}

We have introduced a framework that enables the identification of the important elements in complex nutritional systems, and the quantification of the interactions among them. These interactions include those among the multiple constituents of the ingesta, as well as between behavioural (ingestive) and physiological (post-ingestive) components to nutritional homeostasis. The resulting descriptions provide a powerful means to generate and test hypotheses concerning the mechanisms, ecology and evolution of nutritional systems. We have illustrated this process using data for insects, but the scheme is equally applicable to other animals.

\section{References}

Belovsky GE (1990) How important are nutrient constraints in optimal foraging models or are spatial/temporal factors more important? In Behavioral Mechanisms of Food Selection. NATO ASI Series, vol. 20, pp. 255-278 [RN Hughes, editor]. Berlin: Springer-Verlag.
Bernays EA (1995) Effects of experience on feeding. In Regulatory Mechanisms of Insect Feeding, pp. 279-306 [RF Chapman and $\mathrm{J}$ de Boer, editors]. New York: Chapman and Hall.

Bernays EA \& Chapman RF (1994) Host-Plant Selection by Phytophagous Insects. New York: Chapman and Hall.

Chambers PG, Simpson SJ \& Raubenheimer D (1995) Behavioural mechanisms of nutrient balancing in Locusta migratoria nymphs. Animal Behaviour 50, 1513-1523.

Chapman RF (1995) Chemosensory regulation of feeding. In Regulatory Mechanisms of Insect Feeding, pp. 101-136 [RF Chapman and J de Boer, editors]. New York: Chapman and Hall.

Douglas AE (1994) Symbiotic Interaction. Oxford: Oxford University Press.

Harvey PH \& Pagel MD (1991) The Comparative Method in Evolutionary Biology. Oxford: Oxford University Press.

Hughes RN (editor) (1993) Diet Selection. An Interdisciplinary Approach to Foraging Behaviour. Oxford: Blackwell Scientific Publications.

McNeill S \& Southwood TRE (1978) Role of nitrogen in the development of insect-plant relations. In Biochemical Aspects of Plant and Animal Coevolution, pp. 77-98 [J Harborne, editor]. New York: Academic Press.

Mattson WJ (1980) Herbivory in relation to nitrogen. Annual Review of Ecology and Systematics 11, 119-161.

Mole S \& Waterman PG (1987) Tannic acid and proteolytic enzymes: enzyme inhibition or substrate deprivation? Phytochemistry 26, 99-102.

Pulliam HR (1975) Diet optimization with nutrient constraints. American Naturalist 109, 765-768.

Raubenheimer D (1992) Tannic acid, protein and digestible carbohydrate: dietary imbalance and nutritional compensation in the African migratory locust. Ecology 73, 1012-1027.

Raubenheimer D \& Simpson SJ (1990) The effects of simultaneous variation in protein, digestible carbohydrate and tannic acid on the feeding behaviour of larval Locusta migratoria and Schistocerca gregaria - I: short-term studies. Physiologial Entomology 15, 219-233.

Raubenheimer D \& Simpson SJ (1993) The geometry of compensatory feeding in the locust. Animal Behaviour 45, 953-964.

Raubenheimer D \& Simpson SJ (1994) The analysis of nutrient budgets. Functional Ecology 8, 783-791.

Raubenheimer D \& Simpson SJ (1995) Constructing nutrient budgets. Entomologia Experimentalis et Applicata 77, 99-104.

Raubenheimer D \& Simpson SJ (1996) Meeting nutrient requirements: the roles of power and efficiency. Entomologia Experimentalis et Applicata 80, 65-68.

Raubenheimer D \& Simpson SJ (1997) Integrative models of nutrient balancing: application to insects and vertebrates. Nutrition Research Reviews 10, 151-179.

Raubenheimer D \& Simpson SJ (1998) Nutrient transfer functions: the site of integration between feeding behaviour and nutritional physiology. Chemoecology 8, 61-68.

Raubenheimer D \& Simpson SJ (1999) Integrating nutrition: a geometrical approach. Entomologia Experimentalis et Applicata 91, 67-82.

Schoonhoven LM, Jermy T \& van Loon JJA (1998) Insect-Plant Biology: From Physiology to Evolution. London: Chapman and Hall.

Simpson SJ, McCaffery AR \& Hägele B (1999) A behavioural analysis of behavioural phase change in the Desert Locust. Biological Reviews (In the Press).

Simpson SJ \& Raubenheimer D (1993a) A multi-level analysis of feeding behaviour: the geometry of nutritional decisions. Philosophical Transactions of the Royal Society of London 342B, 381-402. 
Simpson SJ \& Raubenheimer D (1993b) The central role of the haemolymph in the regulation of nutrient intake in insects. Physiological Entomology 18, 395-403.

Simpson SJ \& Raubenheimer D (1995) The geometric analysis of feeding and nutrition: a user's guide. Journal of Insect Physio$\log y$ 41, 545-553.

Simpson SJ \& Raubenheimer D (1996) Feeding behaviour, sensory physiology and nutrient feedback: a unifying model. Entomologia Experimentalis et Applicata 80, 55-64.

Simpson SJ \& Raubenheimer D (1997) The geometric analysis of feeding and nutrition in the rat. Appetite 28, 201-213.

Simpson SJ \& Raubenheimer D (1999a) Geometric models of macronutrient selection. In Neural Control of Macronutrient Selection [H-R Berthoud and RJ Seeley, editors]. Boca Raton, FL: CRC Press (In the Press).

Simpson SJ \& Raubenheimer D (1999b) The hungry locust. Advances in the Study of Behavior (In the Press).

Simpson SJ, Raubenheimer D \& Chambers PG (1995) Nutritional homeostasis. In Regulatory Mechanisms of Insect Feeding, pp. 251-278 [RF Chapman and J de Boer, editors]. New York: Chapman and Hall.

Simpson SJ \& Simpson CL (1992) Mechanisms controlling modulation by haemolymph amino acids of gustatory responsiveness in the locust. Journal of Experimental Biology 168, 269-287.
Stephens DW \& Krebs JR (1986) Foraging Theory. Princeton, NJ: Princeton University Press.

Trumper S \& Simpson SJ (1993) Regulation of salt intake by nymphs of Locusta migratoria. Journal of Insect Physiology 39, 857-864.

Uvarov BP (1966) Locusts and Grasshoppers, vol. 1. Cambridge: Cambridge University Press.

Van Hoven W (1984) Tannins and digestibility in greater kudu. Canadian Journal of Animal Science 64, 177-178.

White TCR (1993) The Inadequate Environment: Nitrogen and the Abundance of Animals. Berlin: Springer.

Zanotto FP, Gouveia SM, Simpson SJ, Raubenheimer D \& Calder PC (1997) Nutritional homeostasis in locusts: is there a mechanism for increased energy expenditure during carbohydrate overfeeding? Journal of Experimental Biology 200, 2437-2448.

Zanotto FP, Raubenheimer D \& Simpson SJ (1994) Selective egestion of lysine by locusts fed nutritionally unbalanced foods. Journal of Insect Physiology 40, 259-265.

Zanotto FP, Simpson SJ \& Raubenheimer D (1993) The regulation of growth by locusts through post-ingestive compensation for variation in the levels of dietary protein and carbohydrate. Physiological Entomology 18, 425-434. 